\title{
Risk of Drowning in People With Parkinson's Disease
}

Key Words: Parkinson's disease; swimming; aquatic therapy; drown

* Correspondence to: Joaquim J. Ferreira, MD, PhD, Laboratório de Farmacologia Clínica e Terapêutica, Faculdade de Medicina de Lisboa, Av. Prof. Egas Moniz, 1649-028 Lisboa, Portugal; E-mail: joaquimjferreira@gmail.com

Relevant conflicts of interest/financial disclosures: The authors declare that there are no financial disclosures or conflicts of interest relevant to this work.

Funding agencies: No specific funding was received for this work. Received: 12 April 2018; Revised: 23 May 2018; Accepted: 1 June 2018

Published online 12 September 2018 in Wiley Online Library (wileyonlinelibrary.com). DOI: 10.1002/mds.27473 
described by $36.5 \%$ of patients $(\mathrm{n}=101)$, and $39.4 \%$

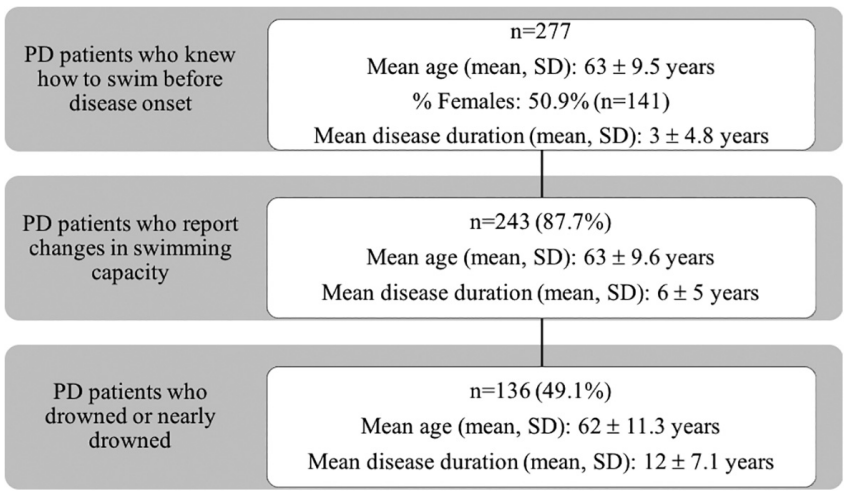

FIG. 1. Frequency and demographic data of PD patients with changes in swimming capacity and drowning events.

Swimming is a common activity, practiced by both healthy and nonhealthy people of all ages. It is a complex activity that requires coordination of breathing with continued and harmonic upper and lower limb movements. ${ }^{1}$ Because of the unique properties of water, aquatic activities are usually associated with facilitated movements and therapeutic properties. ${ }^{2}$

Parkinson's disease (PD) presents with asymmetric motor features that usually interfere with complex activities. ${ }^{3}$ Aquatic therapy is usually considered enjoyable and associated with health benefits in PD patients. ${ }^{2}$ However, although scarce, there is a growing amount of data suggesting swimming difficulties after disease onset. Several reports of patients in Internet forums mention swimming problems, attributing them to mobility problems, lack of coordination, and strength. ${ }^{4}$

This letter refers to an important finding of an online and paper-based self-administered questionnaire, conducted in Portugal and the United Kingdom, to evaluate the frequency of changes in swimming capacity after PD onset. The study was approved by the Centro Académico Medicina de Lisboa (415/16) and the Campus Neurológico Sénior (Ref: 4-2017) Ethics Committee.

An estimated number of 4300 patients was informed about the study. Of these, 381 patients responded to the questionnaire, of whom 72 were excluded because of incomplete data. Participant mean age was 64 years old, with a mean disease duration of 4 years. Tremor was reported as first complaint by $44 \%$ of the patients ( $n=137)$.

Of 309 collected answers, 90\% pf the patients $(\mathrm{n}=277)$ were able to swim before PD symptom onset. Of those who were able to swim, $87.7 \%(n=243)$ noticed a change in swimming performance after disease onset, and $49.1 \%$ ( $\mathrm{n}=136)$ reported having had a drowning or near-drowning episode (Fig. 1). Breathing difficulties during swimming were $(n=109)$ reported the lower limbs as their main cause of difficulty. According to participants' answers, the most frequent reasons for stopping swimming were difficulties in movements coordination $(\mathrm{n}=71)$ and floating $(\mathrm{n}=57)$.

Our results show that PD can seriously interfere with swimming capacity, potentially causing major safety risks. A recent case report describes 4 PD patients with swimming difficulties or drowning after deep brain stimulation surgery. ${ }^{5} \mathrm{We}$ hypothesize that PD patients' swimming difficulties are related to the asymmetric features, lack of coordination, and difficulty performing complex activities. Mean disease duration of patients who reported changes in swimming capacity was $6 \pm 5.0$ years, suggesting an early onset of this difficulty. However, selection bias for patients more capable of accessing the Internet or more motivated to take part in a swimming questionnaire because of previous near-drowning episodes might have occurred. Also, patients' selection has been done based on their assumption of the diagnosis; thereby, it cannot be excluded that patients with atypical parkinsonism were included.

We do not advocate that patients stop swimming or doing aquatic therapy. Instead, we would like to alert to the potential risks associated with swimming and to draw attention to the need to understand the disease-related features that contribute to the changes in swimming performance.

Acknowledgments: We thank Associação Portuguesa de Doentes de Parkinson for its help in distributing the questionnaire.

\section{Maria Ana Neves, ${ }^{1}$ Raquel Bouça-Machado MSc, ${ }^{1,2}$ Daniela Guerreiro, ${ }^{2}$ Verónica Caniça, ${ }^{2}$ and Joaquim J. Ferreira, ${ }^{1,2,3}$ \\ ${ }^{1}$ Instituto de Medicina Molecular, Lisbon, Portugal ${ }^{2}$ Campus Neurológico Sénior (CNS), Torres Vedras, Portugal ${ }^{3}$ Laboratory of Clinical Pharmacology and Therapeutics, Faculty of Medicine, University of Lisbon, Lisbon, Portugal}

\section{References}

1. Conti AA. Swimming, physical activity and health: a historical perspective. Clin Ter 2015;166(4):179-182.

2. Ayán C, Cancela J. Feasibility of 2 different water-based exercise training programs in patients with parkinson's disease: a pilot study. Arch Phys Med Rehabil [Internet] 2012;93(10): 1709-1714.

3. Boonstra TA, Van Vugt JPP, Van Der Kooij H, Bloem BR. Balance asymmetry in Parkinson's disease and its contribution to freezing of gait. PLoS One. 2014;9(7).

4. Parkinson UK [Internet]. Swimming 2017. Available at: https://www. parkinsons.org.uk/forum/thread/53497 